\title{
A REDUCTION OF THE FUNDAMENTAL CONJECTURE ABOUT LOCALLY COMPACT ANR'S
}

\section{A. FATHI AND Y. M. VISETTI}

ABSTRACT. In this note, we show that the conjecture: the product of a locally compact ANR by the Hilbert cube is a Hilbert cube manifold, can be reduced to its compact AR case, i.e.: the product of a compact $A R$ by the Hilbert cube is a Hilbert cube manifold.

1. The school of R. D. Anderson formulated the following fundamental conjecture $[1$, p. 14]:

(C) If $X$ is a locally compact first countable (metrizable) ANR, then $X \times Q$ is a $Q$-manifold. ${ }^{1}$

Recall that a $Q$-manifold is a first countable metrizable space, in which every point has a neighborhood homeomorphic to an open set of the Hilbert cube $Q=I^{\infty}=[-1,+1]^{\infty}$. An affirmative answer to (C) would imply (among other things) the finiteness of homotopy type of compact ANR's [6].

By taking the mapping cylinder of a proper embedding of $X$ into $Q \times$ $[0,1)$, Henderson [4] observed that $(C)$ is implied by:

$\left(C^{\prime}\right)$ If $X$ is a locally compact first countable (me trizable) AR, then $X \times Q$ is a $Q$-manifold.

Our goal is to show that (C) is equivalent to the following particular case (which may be simpler!):

( $\left.C^{\prime \prime}\right)$ If $X$ is a compact metrizable $A R$, then $X \times Q$ is a $Q$-manifold.

We recall that a continuous map is called proper, if inverse images of compact sets are compact sets.

If $X$ is a locally compact space, by $X_{+}$we mean the one point compactification of $X$.

We wish to thank T. A. Chapman for a very useful discussion, which revealed that Lemma 2.1 is the key fact to establish.

Received by the editors February 27, 1974.

AMS (MOS) subject classifications (1970). Primary 58B05; Secondary 54F 40. Key words and phrases. Locally compact ANR, Hilbert cube manifold. [7], [8].

1The analogue for $l^{2}$ in place of $Q$ has recently been proved by Torunczyk 
2. We prove here the key lemma.

Lemma 2.1. Let $Y$ be a locally compact first countable metrizable space, and $X \subset Y$ a retract of $Y$. Then $X \times[0, \infty)$ is a proper retract of $Y$ $\times[0, \infty)$.

Proof. Let $d$ be a metric on $Y$, for which the bounded closed sets are the compact subsets of $Y$. Denoting by $r: Y \rightarrow X$ a retraction of $Y$ on $X$, we define a closed neighborhood $V$ of $X$ by $V=\{x \in Y ; d(r(x), x) \leq 1\}$. Clearly $r \mid V$ is proper.

Choose a proper map $\phi: Y-$ Int $V \rightarrow[0, \infty)$. Then, by observing that $X$ is closed in $Y$, we can use Tietze-Urysohn extension theorem to find an extension $\bar{\phi}$ of $\phi$, such that $\bar{\phi} \mid X=0$.

We then define $\bar{r}: Y \times[0, \infty) \rightarrow X \times[0, \infty)$ by $\bar{r}(y, t)=(r(y), t+\bar{\phi}(y))$. One can easily check that $\bar{r}$ is a proper retraction of $Y \times[0, \infty)$ onto $X \times$ $[0, \infty)$.

Proposition 2.2. If $X$ is a locally compact first countable (metrizable) $A R$, the one point compactification of $X \times[0, \infty)$ is a compact AR.

Proof. $X$ can be embedded as a closed set of $Q \times[0,1)$, and by the AR property, we can consider $X$ as a retract of $Q \times[0,1)$.

If we apply the preceding lemma, we see that $(X \times[0, \infty))_{+}$is a retract of $T=(Q \times[0,1) \times[0, \infty))_{+}$. But $T$ is homeomorphic to the cone on $Q$, hence to $Q$ by $\left[3\right.$, p. 14]. Hence $(X \times[0, \infty))_{+}$is an $A R$, being the retract of the compact AR $Q$.

3. (C') implies $\left(C^{\prime}\right)$.

Proof. Suppos ing that $\left(C^{\prime \prime}\right)$ is true, let $X$ be a locally compact first countable AR. By the preceding proposition, we obtain that $X \times[0, \infty) \times$ $Q$ is a $Q$-manifold. Observe now that $X \times Q$ is a $Q$-manifold, since $X \times$ $[0,1] \times Q$ is the union of two open sets which are $Q$-manifolds, namely $X \times$ $[0,1) \times Q$ and $X \times(0,1] \times Q$.

\section{REFERENCES}

1. R. D. Anderson, T. A. Chapman and R. M. Schori, Problems in the topology of infinite dimensional spaces and manifolds, Mathematische Centrum, Amsterdam, 1971.

2. K. Borsuk, Theory of retracts, Monografie Mat., Tom 44, PWN, Warsaw, 1967. MR $35 \# 7306$. 
3. T. A. Chapman, Notes on Hilbert cube manifolds, University of Kentucky, 1973 (preprint).

4. D. W. Henderson, A simplicial complex whose product with anv ANR is a simplicial complex, General Topology and Appl. 3 (1973), 81-83. MF 47 \#4208.

5. S. T. Hu, Theory of retracts, Wayne State Univ. Press, Detroit, Mich., 1965. MR $31 \# 6202$.

6. L. C. Siebenmann, L'invariance topologique du type simple d'homotopie, d'après T. A. Chapman, Sèminaire Bourbaki, Exposé 428, 1973.

7. H. Torunczyk, Compact absolute retracts as factors of the Hilbert space, Fund. Math. 83 (1973), 75-84.

8. - Absolute retracts as factors of normed linear spaces (preprint).

DÉPARTEMENT DE MATHÉMATIQUES, UNIVERSITÉ DE PARIS-SUD, 91400 ORSAY, FRANCE 